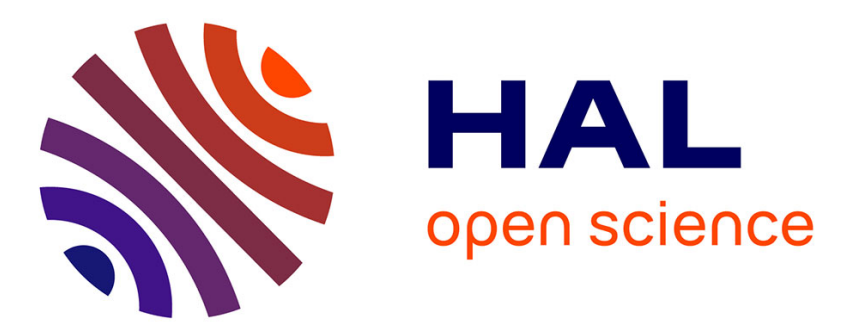

\title{
Field induced coalescence of two free water drops in a viscous dielectric fluid
}

\author{
Jonathan Raisin, Jean-Luc Reboud, Pierre Atten
}

\section{To cite this version:}

Jonathan Raisin, Jean-Luc Reboud, Pierre Atten. Field induced coalescence of two free water drops in a viscous dielectric fluid. ICDL 2011, Jun 2011, Trondheim, Norway. hal-00608051

\section{HAL Id: hal-00608051 \\ https://hal.science/hal-00608051}

Submitted on 12 Jul 2011

HAL is a multi-disciplinary open access archive for the deposit and dissemination of scientific research documents, whether they are published or not. The documents may come from teaching and research institutions in France or abroad, or from public or private research centers.
L'archive ouverte pluridisciplinaire HAL, est destinée au dépôt et à la diffusion de documents scientifiques de niveau recherche, publiés ou non, émanant des établissements d'enseignement et de recherche français ou étrangers, des laboratoires publics ou privés. 


\title{
Field induced coalescence of two free water drops in a viscous dielectric fluid
}

\author{
J. Raisin, P. Atten and J.-L. Reboud \\ G2Elab, (Grenoble Electrical Engineering laboratory) \\ CNRS, Grenoble INP \& Joseph Fourier University \\ Grenoble (France) \\ jean-luc.reboud@grenoble.cnrs.fr
}

\begin{abstract}
The basic process of coalescence of droplets in a flowing water-in-oil emulsion under the action of an electric field is considered. The coalescence probability depends on the ratio of time of close proximity of droplets and time of decrease of spacing down to drops contact. For two free drops aligned with the field, the dynamical problem consists in the deformation of the drops, their motion and the thinning of the oil film between the drops. For very small droplets, assuming a negligible interface deformation, a very small initial spacing and a high value of viscosity ratio leads to an order of magnitude estimate of the time required for the drops to achieve contact. Numerical simulations confirm that this time is roughly inversely proportional to the maximum initial electrostatic pressure $p_{e 0}$ at the facing interfaces and point up an influence of the electric Bond number defined as the ratio of electric and capillary forces.
\end{abstract}

Keywords-component; electrocoalescence; film thinning

\section{INTRODUCTION}

The phenomenon of merging of conducting liquid droplets suspended in an insulating liquid or gas, under the action of an electric field, is called electrocoalescence (EC) [1]. This process operates in electrocoalescers that are used in the petroleum industry for the removal of water coproduced with crude oil in the form of stable emulsions during recovery and desalting operations [2]. EC promotes the increase of the water droplets mean size and facilitates their gravitational separation from the oil phase [2]. However, the control and increase of the electro-coalescers efficiency suffer from the lack of precise knowledge of the numerous processes involved in EC. In particular, in modern electrocoalescers the droplets merging arises from the combined effects of hydrodynamic and electric forces. But, even in the simplest case of water droplets in a pure dielectric fluid, expressions for their rate of coalescence in a flowing emulsion are still missing.

We consider here the basic problem of electrically induced coalescence of two neighboring drops also affected by the liquid flow. For two close immobile water drops anchored on capillary tubes, the field induced electrostatic pressure results in a deformation of the interfaces. Above a critical value of the potential difference, the interfaces disrupt, potentially leading to coalescence $[3,4]$. For two drops which can move freely in a suspending viscous fluid, we do not deal with a stability problem for the static deformed interfaces but with a dynamical problem in which expel of the fluid between the drops plays the major role.

This work was funded by the project "Electrocoalescence - Criteria for an efficient process in real crude oil systems"; co-ordinated by SINTEF Energy Research. Contact person is L.E. Lundgaard. The project was supported by The Research Council of Norway, under the contract no: 169466/S30, and by the following industrial partners: Aker Solutions AS, BP Exploration Operating Company Ltd, Hamworthy Technology and Products AS, Shell Technology Norway AS, Petrobras, Saudi Aramco and Statoil ASA.).

\section{TOWARDS A TIME BASED EC CRITERION}

In the state-of-the-art technologies for dewatering crude oils, EC happens in two stages. Firstly, the turbulent flow induces numerous quasi collisions of the water droplets. Then, the coalescence which would not occur naturally in most cases without applied electric field is promoted by the induced attraction force. A very important step towards the estimation of electrocoalescers efficiency involves the modeling of the time evolution of a flowing emulsion under an electric field. In this event, numerical simulation would be extremely suitable to determine the droplets trajectories and their possible merging. Though, it would require huge computing and (3-D) software capabilities that are, yet, unaffordable. Another possible approach consists in determining the droplets quasi collisions and using a coalescence probability law. The crucial point is then to obtain a criterion stating that the time $t_{\text {prox }}$ of close proximity of two droplets in a flowing emulsion is greater than the time $t_{\text {contact }}$ required for expelling the fluid between them.

As the droplets have a small size (typically tens of micrometers in the emulsions) and as the suspending liquid is much more viscous than water, inertial effects are negligible and, in the absence of electric field, the droplets motion is induced by the local shear flow. As sketched in Fig. 1, two close drops roll around one another with a relative velocity $\sim \dot{\gamma}\left(z_{2}-z_{1}\right), \dot{\gamma}$ being the shear rate and $x_{i}$ and $z_{i}$ denoting the coordinates of the drops centers. For droplets of radius $R_{l}$ and $R_{2}$ there is proximity when $\left|x_{2}-x_{1}\right|<\max \left(R_{1}, R_{2}\right)$ and :

$$
t_{\text {prox }} \sim 2 \max \left(R_{1}, R_{2}\right) /\left[\dot{\gamma}\left(R_{1}+R_{2}+s\right)\right]
$$

( $s$ : spacing between drops). In the case $R_{1} \sim R_{2} \gg s, t_{\text {prox }} \sim$ $1 / \dot{\gamma}$. We now turn toward determining $t_{\text {contact }}$ in the particular case of drops centerline aligned with the electric field.

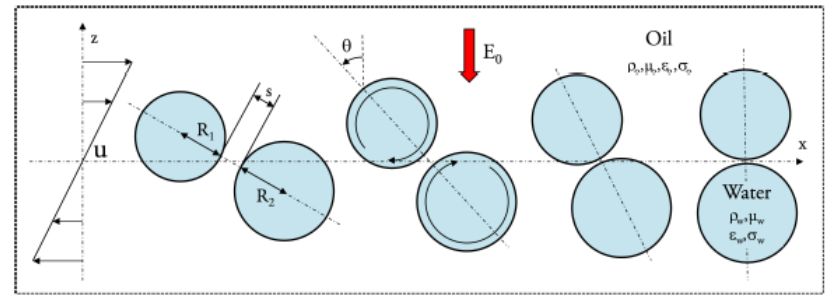

Figure 1. Schematic representation of a collision between two water drops due to the local shear flow. $\theta$ is the angle between the field direction and the drops centerline. 


\section{NUMERICAL SIMULATION}

We consider the time-dependent problem of drainage of the oil film between the water droplets. The film thinning arises from the motion and deformation of the two drops of conducting water, suspended in initially quiescent insulating oil and subjected to a uniform electric field. Simulations have been performed using the commercial software COMSOL Multiphysics $^{\mathrm{TM}}$ which allows Electrohydrodynamic coupling and determination of the liquids flow fields and of the associated drops deformation. The retained configuration is presented in Fig. 2. The computational load is reduced by assuming axial symmetry and, in the particular case of equalsized droplets, by considering the plane of symmetry defined by $z=0$. The electric field is created by imposing a voltage step between two planar electrodes (upper and lower boundaries of the domain) separated by a distance $H$ (Fig. 2 ).

The conducting drops are assumed to be electrically neutral; their potential at any time $t$ is determined by solving the Laplace equation taking into account their shape and position. Gravitational effects are negligibly small for the range of drop diameter investigated. Motion of the fluids is governed by the Navier-Stokes equations, which are solved in oil and water (ensuring the conservation of droplets volume) taking into account the boundary conditions and the interfacial forces (the liquids are assumed to be surfactant free). The main modification of the software concerns the implementation of the interfacial tension forces in axisymmetric case through a weak formulation of the fluid dynamics equations [5]. Deformation of the water/oil interface is performed through an Arbitrary Lagrangian-Eulerian (ALE) formulation with moving meshes. This approach allows us to track the interface deformation in a very accurate way [5].

Simulations were performed considering drops of tap water $\left(\rho_{w}=1000 \mathrm{~kg} / \mathrm{m}^{3}\right.$, dynamic viscosity $\mu_{w}=1 \mathrm{mPa} . \mathrm{s}$, dielectric constant $\varepsilon_{r, w}=80$, conductivity $\sigma_{w} \geq 0.05 \mathrm{~S} / \mathrm{m}$ ) in a polybutene oil $\left(\rho_{\text {oil }}=824 \mathrm{~kg} / \mathrm{m}^{3}, \mu_{\text {oil }}=10.5 \mathrm{mPa} . \mathrm{s}, \varepsilon_{r, \text { oil }}=2.3, \sigma_{\text {oil }} \leq 10^{-12}\right.$ $\mathrm{S} / \mathrm{m}$ ) with interfacial tension $\gamma$ on the order of $2510^{-3} \mathrm{~N} / \mathrm{m}$ (fluids used in experiments [6,7]). For large separation $s_{0}$ between evenly sized drops $\left(R_{1}=R_{2}=R_{0}\right)$, to a first approxim-

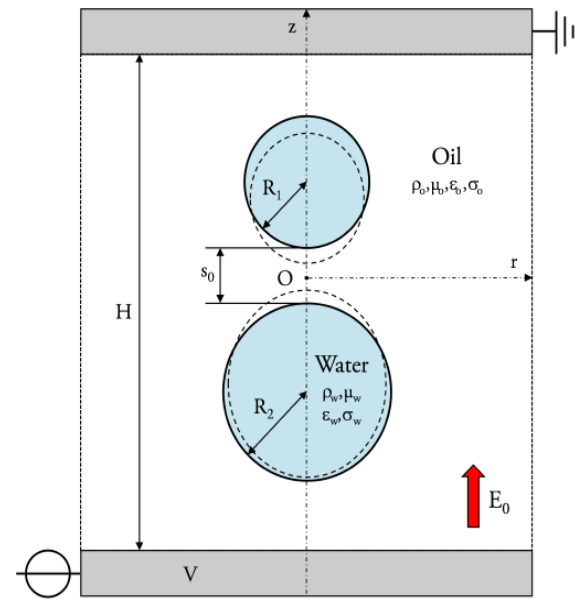

Figure 2. Two conducting drops freely suspended in an insulating oil and aligned with a uniform imposed field (electrode spacing $\mathrm{H}=20 \mathrm{R}_{2}$ ). ation the time necessary for drops to get into contact depends only on the relative spacing $s_{0} / R_{0}$ and not on $R_{0}$ (Fig. 3-a), despite the transient oscillation - accompanying the elongation - that is well marked for big drops (Fig. 3-b). This figure also shows that, at least for the retained applied field, during more than $95 \%$ of the oil drainage time there is no significant elongation and, therefore, no deformation of small droplets.

For large enough initial spacing $s_{0}$, a very rough derivation based on Stokes formula and on the point-dipole approximation for drag and electric forces respectively leads to [8]:

$$
t_{\text {contact }}=\frac{8}{15} \frac{\mu_{\text {oil }}}{\varepsilon_{0} \varepsilon_{r, \text { oil }} E_{0}^{2}}\left[\left(\frac{R_{1}+R_{2}+s_{0}}{R_{1}+R_{2}}\right)^{5}-1\right]
$$

and gives good predictions (within $20 \%$ ) for $s_{0}$ greater than about $R_{0} / 2$ [6]; this is due to under-estimates of both forces which partly compensate. But for $s_{0} / R_{0}$ smaller than about 0.2 , the variation of $t_{\text {contact }}$ versus $s_{0}$ given by (2) is not correct and a different analysis is necessary.

\section{FILM DRAINAGE}

The lubrication theory [9] which leads to drainage times for fully mobile interface much smaller than the computed ones [6] is not relevant because, in the present case, the axial variation of the radial velocity component $u_{\text {oil }}$ in oil does not provide the main contribution to viscous dissipation. A rough estimate of

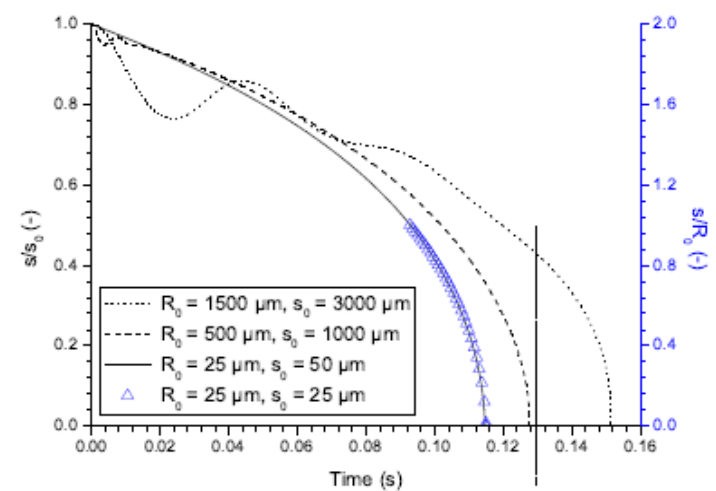

(a)

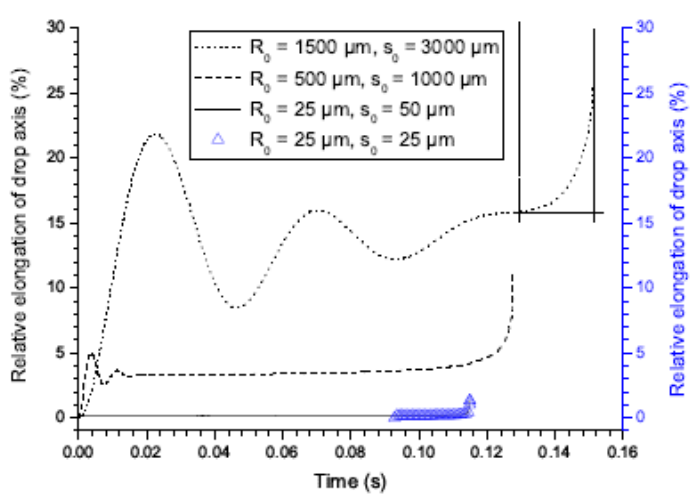

(b)

Figure 3. Temporal evolution of the relative oil film thickness $\mathrm{s} / \mathrm{s}_{0}$ (a), and drops axial elongation (b), for different drops radii $\mathrm{R}_{0}$. The applied field is $E_{0}$ $=3 \mathrm{kV} / \mathrm{cm}$; initial separation $: s_{0}=2 R_{0}$ (or $s_{0}=R_{0}-$ triangle marks). 
the velocity $w_{\mathrm{s}}$ of the drops of same radius $R_{0}$ can be derived by assuming a very small spacing $\left(s<<R_{0}\right)$ and a negligible drops deformation. Denoting by $z_{\mathrm{s}}$ the ordinate of the upper spherical interface and taking for $z_{\mathrm{s}}$ the paraboloidal approximation $z_{\mathrm{s}}(r)=1 / 2\left(s+r^{2} / R_{0}\right)$, from mass conservation we obtain for the mean radial velocity component $\bar{u}_{\text {oil }}$ ( $r$ : radial co-ordinate) :

$$
\bar{u}_{\text {oil }}(r)=w_{\mathrm{s}} r /\left(s+r^{2} / R_{0}\right)
$$

with $\bar{u}_{\text {max }}=(1 / 2) \sqrt{R_{0} / s} w_{s}$ at $r=r^{*}=\sqrt{R_{0} s}\left(r^{*}\right.$ is also the length scale of radial variation of electrostatic pressure $p_{e}$ at interfaces). Neglecting the inertial terms, we deduce from Navier-Stokes equations (cylindrical co-ordinates $r$ and $z$ ) :

$$
\begin{gathered}
\frac{\partial p}{\partial r} \cong \mu_{\text {oil }}\left[\frac{1}{r} \frac{\partial}{\partial r}\left(r \frac{\partial u_{\text {oil }}}{\partial r}\right)-\frac{u_{\text {oil }}}{r^{2}}+\frac{\partial^{2} u_{\text {oil }}}{\partial z^{2}}\right] \\
\frac{\partial p}{\partial z} \cong \mu_{\text {oil }}\left[\frac{1}{r} \frac{\partial}{\partial r}\left(r \frac{\partial w_{\text {oil }}}{\partial r}\right)+\frac{\partial^{2} w_{\text {oil }}}{\partial z^{2}}\right]
\end{gathered}
$$

where $p$ stands for pressure in oil and $w$ for axial velocity component. The flow of oil in the film induces a convective motion in the water drops analogous to a vortex ring with length scale $r^{*}$. In central zone of the oil film the derivatives $\partial u_{\text {oil }} / \partial z$ and $\partial u_{w} / \partial z$ are close to the normal derivatives of velocities at interfaces and the continuity of viscous stress imposes :

$$
\mu_{\text {oil }} \partial u_{o i l} / \partial z=\mu_{w} \partial u_{w l} / \partial z
$$

As $\mu_{o i l} \gg \mu_{w}$ the radial velocity component at interfaces takes a value close to $\bar{u}_{\max }$ (Fig. 4 ) and we obtain the estimates :

$$
\begin{gathered}
\left|\frac{\partial u_{o i l}}{\partial z}\right|\left(r, z= \pm z_{s}\right) \sim \frac{\mu_{w}}{\mu_{o i l}} \frac{\bar{u}_{\text {max }}(r)}{r^{*}} \\
\left|\frac{\partial^{2} u_{o i l}}{\partial z^{2}}\right|=\left|\frac{\partial}{\partial z}\left(\frac{\partial u_{o i l}}{\partial z}\right)\right| \sim \frac{1}{s}\left|\frac{\partial u_{o i l}}{\partial z}\right| \sim \frac{\mu_{w}}{\mu_{o i l}} \frac{w_{s}}{s^{2}}
\end{gathered}
$$

Taking into account the radial variations of $u_{\text {oil }}$ (with length scale $r^{*}$ ), we deduce from (4) the following order of magnitude

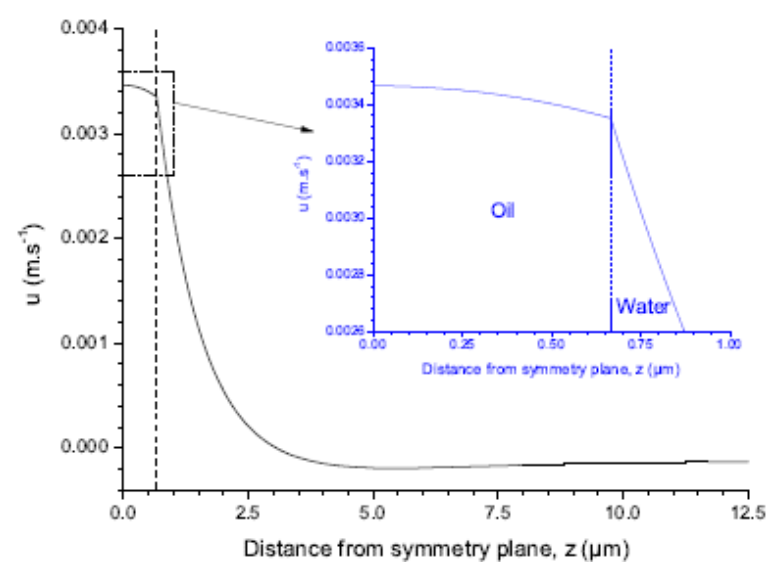

Figure 4. Computed profile of the radial velocity component in oil and water at $r=\left(R_{0} s\right)^{1 / 2}$ and $s=s_{0} / 2$ in the reference case. for the radial derivative of pressure in oil :

$$
\left|\frac{\partial p}{\partial r}\right| \sim \mu_{o i l} \frac{w_{s}}{R_{0}^{1 / 2} s^{3 / 2}}\left(1+\alpha \frac{\mu_{w}}{\mu_{o i l}} \sqrt{\frac{R_{0}}{s}}\right)
$$

where $\alpha$ is a numerical constant. In practice, $s / R_{0}$ is not extremely small (except very close to contact). In the asymptotic case $\left(\mu_{w} / \mu_{o i l}\right)\left(R_{0} / s\right)^{1 / 2}<<1$, we obtain for the pressure difference $(\Delta p)_{r}$ :

$$
(\Delta p)_{r}=p(0,0)-p_{\infty} \sim\left|\frac{\partial p}{\partial r}\right| r^{*} \sim \mu \frac{w_{S}}{s}
$$

Balancing $(\Delta p)_{r}$ with the maximum electrostatic pressure $p_{e 0}$ leads to an estimate for $w_{s}$. Taking Davis expression $p_{e 0}=$ $\varepsilon_{\mathrm{r}, \text { oil }} \varepsilon_{0}\left(E_{3} E_{0}\right)^{2} / 2$ [10] corresponding to maximum field at the droplets surface and a fitted power law $1.87\left(R_{0} / s\right)^{0.85}$ for the variation of $E_{3}$ [6], leads to the expression (with a factor $\beta$ ) :

$$
w_{\mathrm{s}} \sim \frac{1.75}{\beta} \frac{\varepsilon_{0} \varepsilon_{r, o i l} E_{0}{ }^{2}}{\mu_{\text {oil }}} \frac{R_{0}{ }^{1.70}}{s^{0.70}}
$$

The temporal evolution of the spacing $s$ then is :

$$
s(t)=s_{0}\left[1-\frac{2.975}{\beta} \frac{\varepsilon_{0} \varepsilon_{r, o i l} E_{0}^{2}}{\mu_{\text {oil }}}\left(\frac{R_{0}}{s_{0}}\right)^{1.7} t\right]^{1 / 1.7}
$$

Fig. 5 shows that (12) very well accounts for the decrease of spacing obtained by simulation. The contact time is :

$$
t_{\text {contact }}=\frac{\beta}{2.975} \frac{\mu_{\text {oil }}}{\varepsilon_{0} \varepsilon_{\text {r,oil }} E_{0}^{2}}\left(\frac{s_{0}}{R_{0}}\right)^{1.7}=\frac{\beta}{1.7} \frac{\mu_{\text {oil }}}{p_{e 0}}
$$

\section{RESULTS OF SIMULATION}

As the primary interest of electrocoalescence is to resolve the water-in-oil emulsions, we considered water droplets of small size (tens of micrometers) with an initial spacing $s_{0} \sim 1$ $\mu \mathrm{m}$ and a polybutene oil (see [7]); the reference case is relative to $R_{0}=20 \mu \mathrm{m}, s_{0}=1 \mu \mathrm{m}$. A systematic study was conducted by varying the applied field. Firstly, simulations show that the small drops deformation is of very limited amplitude (Fig. 3) and very regular [6], thus supporting the hypothesis of §IV on non deformation of droplets.

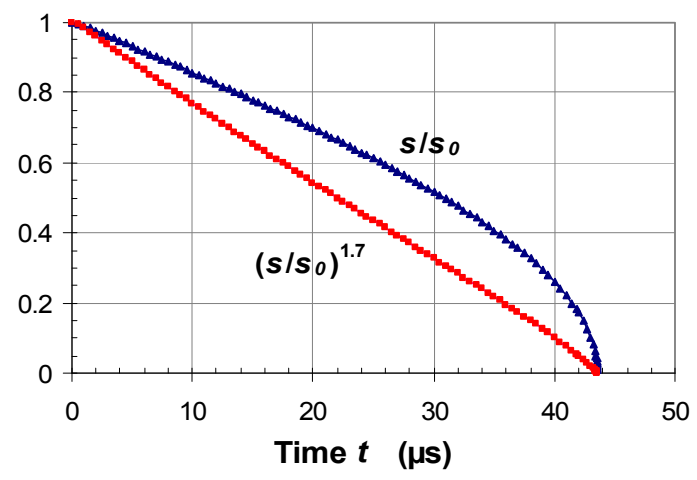

Figure 5. Decrease of the spacing $s$ versus time in the reference case $\left(R_{0}=20 \mu \mathrm{m}, s_{0}=1 \mu \mathrm{m}\right)$ and $E_{0}=4 \mathrm{kV} / \mathrm{cm}$. 
The most striking result is that the contact time: $t_{\text {contact }}$ is inversely proportional to the initial maximum electrostatic pressure $p_{e 0}$ (Figs. 6-8). For a given $p_{e 0}$, to a very good approximation, it takes the same value independently of the drops radius and of the initial spacing (Fig. 6).

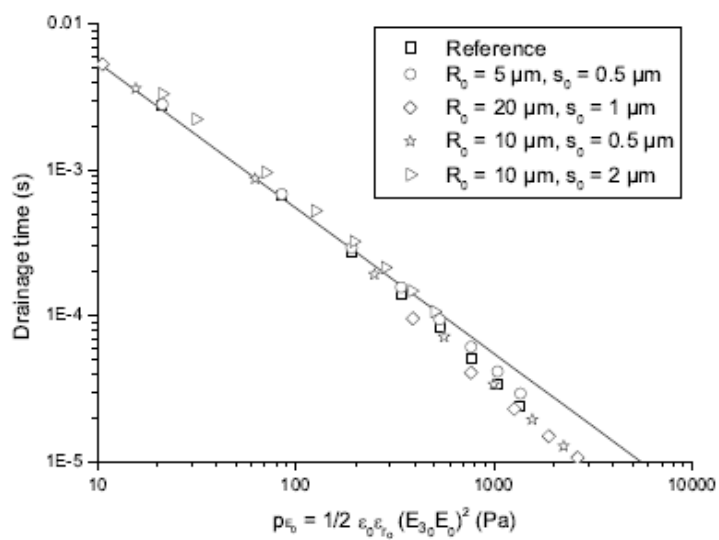

Figure 6. Variations of the drainage time as a function of the initial maximum electrostatic pressure $p_{E 0}$ for even drops (for different $R_{0}$ and $s_{0}$ values). The full line corresponds to (13) with $\beta=8$.

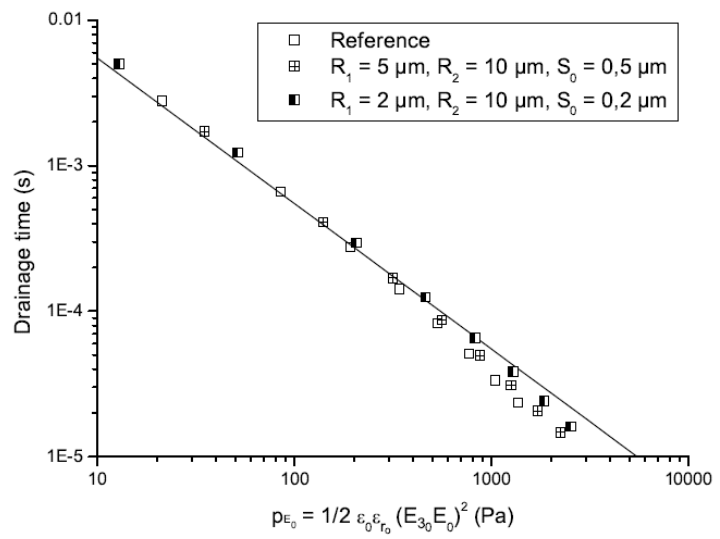

Figure 7. Variations of the drainage time for drops of different radii $R_{I}$ and $R_{2}$. The full line corresponds to (13) with $\beta=8$.

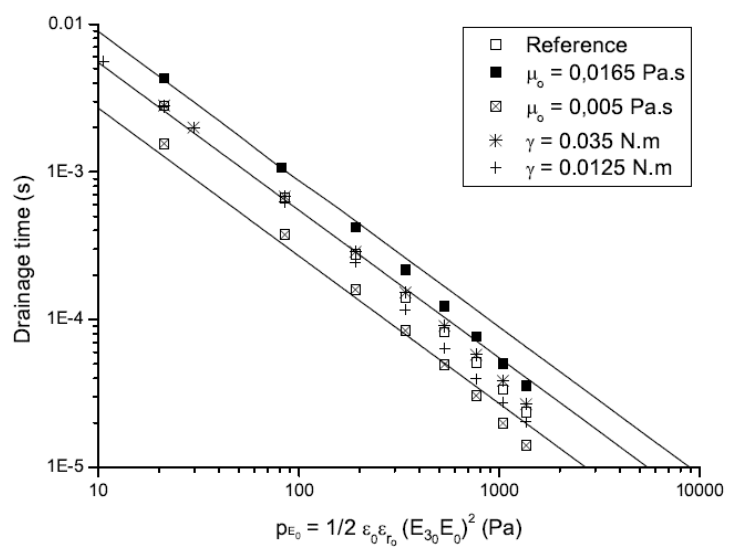

Figure 8. Variations of drainage time as a function of $p_{e 0}$ for even drops of radius $R_{0}$ illustrating the influence of interfacial tension $\gamma$ and viscosity $\mu_{o i l}$. Full lines correspond to (13) with the three $\mu_{o i l}$ values.

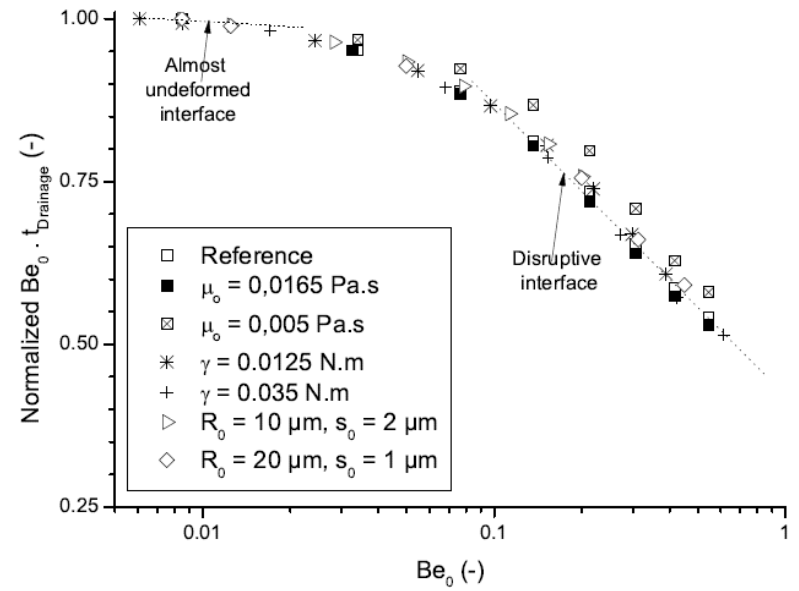

Figure 9. Variations of the normalized product of the electric Bond number $B e_{0}$ by the drainage time $t_{\text {contact }}$ as a function of $B e_{0}$ for even drops.

Moreover, with drops of different size, the contact time has a very weak dependence on the radii ratio (Fig. 7). This behaviour fairly well corresponds to the one predicted by (13). For small to moderate $p_{e 0}$ values $(<200$ to $300 \mathrm{~Pa})$, clearly $t_{\text {contact }}$ increases with viscosity but is independent of the interfacial tension (Fig. 8). For high initial electrostatic pressure, the contact time takes values lower than the one given by (13). In order to understand why this decrease occurs we plotted the product of contact time and electric Bond number $B e_{0}\left(=p_{e 0} R_{0} / \gamma\right)$, normalized by its value for low $p_{e 0}$, as a function of $\mathrm{Be}_{0}$; a universal curve is obtained (Fig. 9), which suggests that strong enough electrostatic pressure might interplay with capillary pressure and slightly elongate the drops, thus facilitating the oil drainage and reducing $t_{\text {contact }}$.

\section{REFERENCES}

[1] L. Lundgaard, G. Berg, S. Ingebrigsten, P. Atten, "Electrocoalescence for oil/water separation: Fundamental aspects" in Emulsions and emulsion stability, vol. 132, pp. 549-592, CRC Press, 2006.

[2] O. Urdahl, N.J. Wayth, H. Fordedal, T.J. Williams, A.G. Bailey, "Compact electrostatic coalescer technology" in Encycl. handbook of emulsion techn., J. Sjöblom, Ed., pp. 679-694, M. Dekker, 2001.

[3] J. Raisin, J.-L. Reboud, P. Atten, "Electrocoalescence of two water drops in oil: Experiment and modelling", Int. J. Plasma Environ.Sci. Techno., vol. 3 (2), pp. 127-132, 2009.

[4] P. Atten, F. Aitken, "Electrocoalescence criterion for two close anchored water drops and estimate for pairs of drops in a field", IEEE Trans. Ind. Appl., vol. 46 (4), pp. 1578-1585, 2010.

[5] J. Raisin, J.-L. Reboud, P. Atten, "Electrically induced deformations of water-air and water-oil interfaces in relation with electrocoalescence", $J$. Electrostatics, in press, 2011.

[6] J. Raisin, "Electrocoalescence in water-in-oil emulsions: towards an efficiency criterion", PhD thesis, Grenoble University, 2011.

[7] J. Raisin, P. Atten, JL. Reboud, 2011, "Electrocoalescence of water drops in oil shear flow: development of experimental set up", ICDL 2011, 26-30 june 2011, Trondheim, Norway

[8] P. Atten, "Electrocoalescence of water droplets in an insulating liquid", J. Electrostatics, vol. 30, pp. 259-270, 1993.

[9] R.H. Davis, J.A. Schonberg, J.M. Rallison, "The lubrication force between two viscous drops", Phys. Fluids A, vol. 1 (1), pp. 77-81, 1989.

[10] M.H. Davis, "Two charged spherical conductors in a uniform electric field : forces and field strength", Quart. J. Mech. Appl. Math., vol. 17, pp. 499-511, 1964. 